\title{
Task- and Time-Dependent Memory Enhancement by Dehydroepiandosterone in Day-Old Chicks
}

\author{
A.N.B. Johnston and P.V. Migues \\ Brain and Behavior Research Group, The Open University, Milton Keynes MK7 6AA, UK
}

\begin{abstract}
We have previously reported the presence of dehydroepiandosterone (DHEA) in the dayold-chick brain, and a role for it in enhanced memory formation. Here we confirm that intracerebral injections of DHEA $5 \mathrm{~min}$ before training on the weak passive avoidance task enhanced recall 24 hours after training. Recall per se on an appetitive visual categorization task was not altered by administration of DHEA $5 \mathrm{~min}$ before training. However administration of DHEA 5 min before limited or very limited training on a visual categorization task (20 or 10 pecks only) appeared to enhance consolidation of this task at test $24 \mathrm{~h}$ after training; reducing the latency and total time taken to complete the test (60 pecks), while not detrimentally altering accuracy. Moreover, DHEA is unlikely to induce this effect via possible anxiolytic effects because it did not alter behavior in the open field test. We also examined diffusion of DHEA throughout the brain at various stages following intracerebral injection.
\end{abstract}

Reprint request to: Amy Johnston, Dept. of Biological Sciences, The Open University, Walton Hall,, Milton Keynes, MK7 6AA, UK; e-mail: a.n.b.johnston@open.ac.uk

Abbreviations: DHEA-S, dehydroepiandosterone-sulphate; DMSO, dimethyl sulphoxide; GABA, gamma-aminobutyric acid; IMHV, intermediate medial hyperstriatum ventrale; LPO, lobus parolfactorius; MeA, Methylanthranilate ; NMDA, Nmethyl-D-aspartate; PREG, pregnenolone

\section{KEYWORDS}

neurosteroids, passive avoidance learning, DHEA, avian, visual discrimination

\section{INTRODUCTION}

The production of significant quantities of neurosteroids and steroids like dehydroepiandosterone (DHEA), pregnenolone (PREG), and their sulphate esters (Baulieu, 1997; Corpechot, 1981; Corpechot et al., 1983; Lanthier \& Patwardhan, 1986), which are synthesized de novo in the brain from cholesterol (Robel \& Baulieu, 1995), has been documented in a wide range of mammalian species (Akwa \& Baulieu, 1999). PREG and DHEA have also been detected in avian species like adult male Japanese quails (Tsutsui \& Yamazaki, 1995) and day-old chicks (Migues et al., 2002). Moreover, in day-old chicks, DHEA is highly concentrated in a brain region known to be crucial for consoli-dating certain early forms of memory, including passive avoidance learning (Rose, 2000) and filial imprinting (Horn, 1991; Johnston et al., 1995). In this region, the intermediate medial hyperstriatum ventrale (IMHV), the levels of DHEA are almost 10-fold higher than those in the forebrain as a whole and more than twice those found in other subregions of the chick forebrain, even though plasma concentrations in chicks are of the same order of magnitude as those found in humans (Lanthier \& Patwardhan, 1986) and rats (Robel et al., 1999; Migues et al., 2002). Such a high concentration of 
DHEA in the IMHV region, which is extremely important for various forms of early neural plasticity in chicks, is suggestive of a direct role of DHEA in such plasticity, but can also simply indicate high levels of activity in this region. Given our previous finding that DHEA is implicated in the consolidation of passive avoidance learning, this hypothesis seemed to warrant further investigation.

DHEA and DHEA-sulphate (DHEA-S) are indeed promising candidates for plasticity-related effects in the central nervous system (Baulieu \& Robel, 1996). These compounds have been shown to increase neuronal excitability by modulating the activity of GABA and sigma receptors (Majewska et al., 1990; Demirgoren et al., 1991; Monnet et al., 1995) and to stimulate neurite growth and synaptic formation during development (Bologa et al., 1987; Roberts et al., 1987; Compagnone \& Mellon, 1998); events that also have been implicated in the active consolidation of various forms of memory in chicks and various rodents (Farkas \& Crowe, 2000; Izquierdo \& Medina, 1997, Rose \& Stewart, 1999). Moreover, behavioral pharmacological studies using several learning and memory models have strongly suggested that DHEA and DHEA-S play a role in the consolidation of memory (Vallee et al., 2001).

DHEA and DHEA-S improve retention in various 'aversive' tasks in rodents, particularly aged rodents (for review see Vallee et al., 2001), including passive and active footshock avoidance (Flood et al., 1988; Reddy \& Kulkarni, 1998a), elevated plus maze (Reddy \& Kulkarni, 1998b), and spatial tasks like the T-maze (Melchior \& Ritzmann, 1996). Moreover, we recently showed that DHEA is involved in the consolidation of a passive avoidance task in chicks (Migues et al., 2002). Such tasks are known to be inherently stressful, however, elevating endogenous corticol/corticosterone (McGaugh, 1989; Cordero et al., 1998). Moreover, performance at test on these tasks in chicks and rodents is also altered by the administration of specific levels of 'stress' hormones like corticosterone, as is the 'weak' version of passive avoidance learning (Sandi \& Rose, 1994; Johnston \& Rose, 1998; McGaugh, 1989). The endogenous levels in the brain but not in plasma, of certain neurosteroids, including a precursor of DHEA (PREG), are directly related to acute stress responses (Barbaccia, 1996a,b); and levels of PREG, not DHEA, correlate with memory performance in rats (Vallee et al., 2001). Nevertheless, unique thus far among the neurosteroids, DHEA has yet to be shown to be specifically altered by exposure to stressful events, suggesting that DHEA may not be directly evoked by stress. Thus, its putative antiamnestic features may relate less to anxiolytic properties and more directly to the biochemical mechanisms of memory consolidation.

Information about the role of the neurosteroids in the consolidation of appetitive tasks, as opposed to aversive tasks, is very limited in any species. Whereas in mice, PREG-S can overcome amnesia induced by an NMDA receptor antagonist in a classic operant conditioning task, it does not, when administered alone, alter behavior on this task. Melchior and Ritzamn (1996) investigated the effect of PREG, PREG-S, DHEA, and DHEA-S on spatial working memory in adult male mice, using a win-shift foraging paradigm in a T-maze, in which an appetitive reward (milk) was offered. The authors found that DHEA, DHEA-S, PREG, and PREG-S $(0.05 \mathrm{mg} / \mathrm{kg}$ i.p. each) given $30 \mathrm{~min}$ before training enhanced memory. Despite their own previous findings, however, that DHEA had an anxiolytic effect whereas PREG-S has a dosedependent anxiogenic effect (Melchior \& Ritzman, 1994), the authors did not investigate the possible differential effects of these neurosteroids on nonspecific behavioral strategies or on anxiety per se.

Most behavioral studies using DHEA or related neurosteroids have been done with male animals. We have previously shown that, at least in chicks, 
behavioral (even learning-related) responses to stressors are often more elevated in male than in female animals (Rogers, 1995; Johnston \& Rose, 1998). DHEA, as opposed to the other neurosteroids, has not been found to show genderdependent effects on memory tasks. Although lack of such a finding cannot be taken to indicate a true lack of such a difference, we have not found any gender-linked differences in the effect of DHEA treatment on memory retention (Migues et al., 2002). Yet, possible anxiolytic effects of DHEA in chicks cannot be dismissed and clearly warrant investigation, such as examining its effects on the open field test (Andrew, 1991).

Bead floor (or pebble floor) learning is a visual categorization learning task commonly used in the chick. This task relies on chicks learning, within the space of a limited number of pecks (usually 60 pecks), to attend selectively to food grains scattered among beads or pebbles that are glued to the floor (Rogers, 1995; Tiunova et al., 1996). Thus, the task simulates a possible 'reallife' scenario, in which chicks rapidly and with limited experience learn to classify objects into food and non-food items after encountering only a few examples of such objects, based on the acquisition of a generalized response to classes of object (beads/grains). This task relies on a food search strategy that is apparently quite rapidly adapted to laboratory-based studies like this and has been used to describe various correlates of memory (Tiunova et al., 1998).

The focus of the current study was to examine the relative memory-enhancing effects of DHEA in an aversive and in an appetitive task in the same species at relatively similar ages. Our aim was to confirm our previous observation that DHEA did indeed facilitate the weakly aversive version of the one-trial passive avoidance task (Experiment 1) and to examine the effects of administering DHEA into the IMHV region on an appetitive task (Experiments 2,3). Various measures of possible non-specific effects of DHEA on behavior, which might influence apparent 'memory' performance accompanied these tests to specifically examine whether DHEA has anxiolytic effects on chicks by testing age- and batch-matched treated chicks on an open field and response to novel object test (Experiment 4). Previous data were acquired from experiments in which neurosteroids were injected into the intermediate medial hyperstriatum ventrale (IMHV) region. Because we considered it important to examine the extent of diffusion of DHEA after such injections, we also completed an experiment using radiolabeled DHEA to examine these parameters (Experiment 5).

\section{MATERIALS AND METHODS}

Animals

Fertile Ross Chunky eggs were incubated in a commercial communal brooder maintained on a 8:16 h light-dark cycle at $37-38^{\circ} \mathrm{C}$. Eggs were candled on day-16 of incubation, and the fertile eggs were transferred to a hatching incubator maintained on a 12:12 $\mathrm{h}$ light-dark cycle at 37$38^{\circ} \mathrm{C}$ the following day. The chicks were allowed to hatch normally and, about $6 \mathrm{~h}$ after hatching, were placed in a group cage maintained on a 12:12 $\mathrm{h}$ light-dark cycle, at $27-29^{\circ} \mathrm{C}$ with chick starter crumbs and water provided ad libitum. All animal experimental work was carried out under personal and project Home Office Licenses, according to the U.K. Animals (Scientific Procedures) Act 1986.

\section{Administration of DHEA:}

DHEA (Sigma, UK) was dissolved in dimethylsulphoxide (DMSO, Sigma, UK) to create a stock solution of $0.1 \mathrm{M}$ and then diluted with $0.9 \%$ sterile saline to the appropriate concentration for injections. The final concentration of DMSO in all 
solutions was less than $0.001 \%$. DHEA ( 3 or $30 \mathrm{ng}$ per chick, equivalent to 10 or $100 \mathrm{pmol})$, or saline containing less than $0.001 \%$ DMSO (control) were injected intracerebrally, 5 microliters per hemisphere into both hemispheres, $5 \mathrm{~min}$ before training. The solutions were injected using a Hamilton microsyringe fitted with a plastic sleeve as a stop, allowing an injection depth of $3 \mathrm{~mm}$. A Plexiglass head holder was used to direct the injection into both the right and left intermediate and medial hyperstriatum ventrale (IMHV). The injection needle was left in place for $5 \mathrm{~s}$. Methylene blue stain was used in preliminary trials to confirm that the headholder was calibrated and that the injections were going to the IMHV region. Moreover, at the conclusion of each experiment, the brains were dissected and a visual inspection of the termination site of each injection was made. Less than $2 \%$ of the injections did not appear to terminate in the IMHV. In such cases, the data were not included in the analysis.

\section{Behavioural testing}

Passive avoidance learning: Memory retention was studied using the weak passive avoidance task (Sandi \& Rose, 1994; Burne \& Rose, 1997; Crowe \& Hamalainen, 2001). One-day-old chicks were placed in pairs into aluminium pens illuminated by red $25 \mathrm{~W}$ light bulbs, and chick crumbs were scattered on the floor. After at least $1 \mathrm{~h}$ of acclimatization, the chicks were pre-trained by presenting them with a small ( $3.5 \mathrm{~mm}$-diameter) white bead for $10 \mathrm{~s}$. We repeated this procedure 3 times with an interval of at least 5 min between presentations. Chicks that failed to peck in at least two of the three trials were excluded from subsequent treatment. Five min after pretraining, the chicks were trained by a $30 \mathrm{~s}$ presentation of a 4-mm shiny chrome bead coated with $10 \% \mathrm{MeA}$ in ethanol. Twenty-four $h$ after training, the chicks were tested with a $10 \mathrm{~s}$ presentation of a dry $4-\mathrm{mm}$ chrome bead, followed ( $5 \mathrm{~min}$ later) by a $10 \mathrm{~s}$ presentation of a white bead (the discrimination trial). We trained and tested each chick only once. After the test, the chicks were killed, and the site of injection was confirmed. Chicks that showed discrimination at test; namely, avoided the chrome bead but pecked at the white bead, were classified as able to recall the task, whereas those that pecked at both beads at test were regarded as amnesic. Whereas clearly some chicks may remember the task and avoid both beads during the test, the standard criteria we use to indicate recall is that the chicks show a specific memory of the aversive properties of the chrome bead and do not generalize to the white bead (Burne \& Rose, 1997). Failure to meet this basic criterion results in exclusion from further analysis. By contrast, chicks that peck at the white bead during testing demonstrate that their general pecking behavior is relatively unaffected by training and that no significant motor or visual impairment has resulted from treatment. Thus, we included in the final analysis only chicks that (a) in at least two of three trials pecked during pre-training, (b) pecked at the chrome bead during training, and (c) pecked at the white bead during the test.

The results were expressed as percent avoidance, a group level measure of recall calculated as follows.

no. chicks that pecked at white bead but not at chrome bead at test no. chicks that reached all criteria

\section{Beadfloor}

Soon after hatching, the chicks were placed, either in pairs (Experiment 2) or individually (Experiment 3), into $20 \times 25 \times 20 \mathrm{~cm}$ aluminum cages, illuminated with red $25 \mathrm{~W}$ light bulbs and were provided with food and water ad libitum. The chicks remained in these pens but were handled. and the pens were cleaned at least twice daily, for $2 \mathrm{~d}$. The experiments began $3 \mathrm{~d}$ after hatching. 
Food was removed from the home cage for 2 to $3 \mathrm{~h}$ before training. The chicks were individually placed into the experimental (training/testing) cage, which was identical to the home cage except for a transparent perspex front wall and, in Experiment 2, a mirror that was stuck to the rear wall of the cage. The perspex floor of the 'test' cage was covered in eight different colored beads, glued to the floor in twelve rows of fifteen beads each. Before training and testing, around $2 \mathrm{~g}$ of standard chick starter crumbs were scattered on the floor among the beads.

During training, each chick remained in the experimental cage until it had completed either 20 pecks (Experiment 2; limited training) or 10 pecks (Experiment 3; very limited training) at objects on the floor. We scored repeated pecks at the same object, not interrupted by pecks at other objects, as a single peck. We scored all data manually, using an adapted computerized event recorder. Pecks were scored as 'correct' (peck at a food grain) or 'error' (peck at bead). We also recorded defecations, scratching, grooming, and jumping. Immediately after completing its allocated number of pecks, each chick was returned to the home cage, where it remained until testing $24 \mathrm{~h}$ later.

The housing conditions were changed between Experiment 2 and Experiment 3 (paired housing or individual housing) in an attempt to remove the requirement for a mirror ('pseudo-social companion') during training and testing. The mirror seemed to attract a great deal of attention and to increase latency and pecking times unnecessarily (see Figs. $3 \& 5$ ). Using individual housing for several days before training/testing (as per Rogers, 1995) successfully overcame isolation stress in these social animals. Indeed, the behavioral parameters associated with the bead-floor test and its 'parent' have been widely investigated (see Rogers, 1995) and thus, a great deal of confidence can be placed in specific effects of subtle variations in housing, training, and testing conditions.
We conducted testing and training in the same manner but in testing, the chicks remained in the experimental cage until they had completed 60 pecks at objects on the floor. In both Experiments 2 and 3, we allowed a maximum time of $600 \mathrm{~s}$ to complete training and a maximum time of $300 \mathrm{~s}$ to complete the test.

\section{Open field and response to novel object tests:}

Open field and response to novel object tests were conducted on chicks from the same batches as those used in the very limited beadfloor tests. The chicks were housed in pairs (to match those used in Experiment 2) for several days before testing. One chick in each pair, the target chick, was injected with DHEA (30 ng) or saline $5 \mathrm{~min}$ before testing or $24 \mathrm{~h}$ before testing (to match the times of injection pre-training and effects at testing $24 \mathrm{~h}$ later). The chicks were returned to their home cage after testing.

For the open field test, we used a 1-m square gray non-reflective plastic arena. The floor was covered in the same paper toweling that was used in the chick's home cage. The toweling was divided into $10-\mathrm{cm}$ square sections. Each target chick was placed, along with its companion chick, into the arena at the same start point ('top' left corner). We videotaped the chick's behavior during the $300 \mathrm{~s}$ test and simultaneously scored the behavior, using a computerized event recorder. We recorded the number of squares crossed, defecations, bouts of peeping, grooming, and scratching. Immediately following the end of the $300 \mathrm{~s}$ open field test, the chicks were removed from the open field, a novel object (blue and white box, 3.5 square centimeters with 2 red spots on each side) was placed into one marked square, and the chicks were returned to the new 'start square' (middle left) in the open field. Again, we videotaped the test and simultaneously scored the behavior of the target chick, using a computerized event recorder. 
The number of squares crossed, defecations, bouts of peeping, grooming, and scratching were noted. Also noted were approaches toward or contact with the novel object, time spent in the same square as the novel object, and time spent in a square adjacent to the novel object, using playback analysis.

With all experiments, the contribution of each batch to each group was balanced as much as possible, given the inevitable variations in hatch size and exclusion because of failure to meet the criteria. Each chick was trained and tested once only and on only one behavioral task (except open field and response to novel object tests, which we conducted using the same 'target' chick).

\section{Statistical analysis:}

Statistical comparisons between the percentage avoidance scores of the groups were performed using the G-test (Sokal \& Rohlf, 1981). We also compared the numbers of chicks excluded from each group, due to failure to reach criteria, where appropriate, by G-tests. We pooled the results from male and female chicks as data from earlier experiments indicated no gender difference in the avoidance response to DHEA. Scores from the beadfloor tests (errors) were grouped into blocks of 20 (Experiment 2/3) or 10 (Experiment 3) and compared using Kruskal-Wallis tests (Sokal \& Rohlf, 1981). Post-hoc tests were conducted using Mann-Whitney U-tests. 'Timing' data (latency, total time, time spent pecking) was compared using the one-way ANOVA and the post-hoc Tukey's honestly significant difference tests. We recorded insufficient numbers of defecations, grooming bouts, jumps, and scratching for further analysis.

We used ANOVA to compare the number of squares crossed, the number of bouts of peeping, and latency to move out of the start square in both the open field and the response to novel object tests, using the factor treatment (DHEA or saline pre-training, DHEA or saline post-training). We recorded insufficient numbers of grooming bouts, jumps, and scratching for further analysis. As most chicks defecated once in each test, in each group the number of chicks that defecated were compared using G-tests.

\section{Diffusion experiment}

Chicks were injected with 3 nanograms of $\left[{ }^{3} \mathrm{H}\right]$ DHEA into the IMHV $(112,000 \mathrm{dpm})$. At various times after injection $(5,15,30,60 \mathrm{~min})$, the birds were killed and the brain was removed. The IMHV and the lobus parolfactorius (LPO) were dissected from each brain, weighed, and frozen on dry ice. The rest of the brain, including the remaining forebrain and the mid-hindbrain, was also weighed and frozen on dry ice. Each sample was then homogenized using a Polytron homogenizer (Kinematica $\mathrm{GmbH}$, Switzerland) at 27,000 rpm with cold phosphate-buffered saline (PBS) $(1: 10)$. The composition of the buffer $(\mathrm{mM})$ was as follows: $\mathrm{CaCl}_{2} .2 \mathrm{H}_{2} \mathrm{O} 0.9, \mathrm{MgCl} .6 \mathrm{H}_{2} \mathrm{O} 0.5, \mathrm{KCl}_{2}$ 2.6, $\mathrm{KH}_{2} \mathrm{PO}_{4} 1.5, \mathrm{NaCl} 136.0, \mathrm{Na}_{2} \mathrm{PO}_{4} 8.1(\mathrm{pH}$ 7.4). An aliquot (100 microliter) from each homogenate was placed in a scintillation vial, mixed with $5 \mathrm{~mL}$ of scintillation liquid (Pico Fluor $^{\mathrm{TM}}$ 15, Packard Bioscience B.V., The Netherlands). Radioactivity was counted in a Beckman LS 1701 liquid scintillation counter.

\section{RESULTS}

\section{Experiment 1: Passive avoidance tests}

Mirroring our previous results, when $30 \mathrm{ng}$ of DHEA/chick was injected $5 \mathrm{~min}$ before training, we found a significant enhancement of avoidance at test compared with the performance of matched saline-treated chicks $(G=4.12, P<0.05$; Fig. 1). 


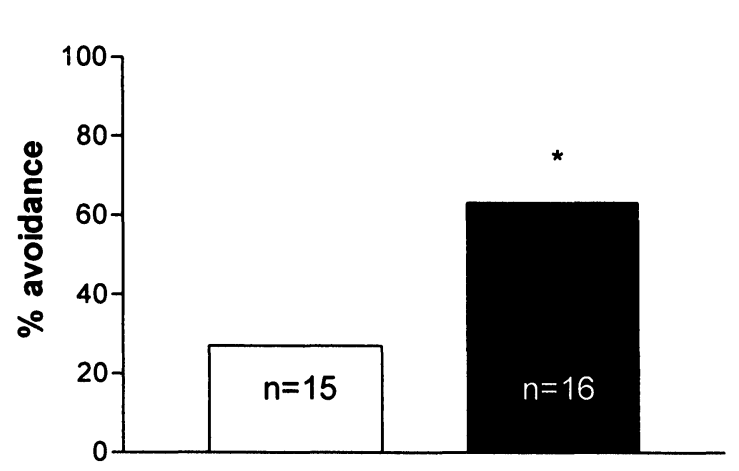

Fig. 1: Effects of bilateral pretraining injection of DHEA (30 ng; solid bar) compared to saline (open bar) on recall $24 \mathrm{hr}$ after training using the 'weak' version of the passive avoidance task (with $10 \% \mathrm{MeA}$ ). Results are expressed as percentage avoidance from each group. *Avoidance tested at $24 \mathrm{~h}$ posttraining is significantly enhanced by DHEA $(P<0.05)$. $n$-values are given in the appropriate bars.

This result was unlikely to be due to the nonspecific effects of treatment with DHEA on training because an equivalent proportion of each group did not peck during training $(2 / 19$ saline treated chicks versus $1 / 21$ DHEA treated chicks, $(G=0.48, P>0.25)$. The result is equally unlikely to be due to nonspecific effects on attention or other non-specific behaviors during testing because similar proportions of both DHEA- and saline-treated chicks did not discriminate $(2 / 17$ saline-treated versus 3/19 DHEA-treated chicks). Overall, 12 of 43 chicks (28\%) initially placed into home cages were excluded from the analysis. Thus, the results of the present study extend our previous data suggesting an enhancing effect of 30 nanograms DHEA per chick, administered bilaterally on avoidance responses following 'weak' training. It is interesting that many of the DHEA-treated chicks shook their heads, .peeped, and backed away from the test bead, indicative of a memory of the aversive nature of the 'bittertaste' previously associated with this bead.

\section{Experiment 2: Beadfloor testing chick, limited training}

There was no significant difference between either group of DHEA-treated and saline-treated chicks $(G=0.25, P=0.88)$ in the number of errors in the training block of 20 pecks. As evident in Fig. 2, all chicks showed similar pecking at beads (errors) and grains. The treatment also had no significant effect on pecking in either the first $(\mathrm{G}=4.74, \mathrm{P}=0.09)$, second $(\mathrm{G}=0.52, \mathrm{P}=0.77)$ or third $(\mathrm{G}=0.24, \mathrm{P}=0.89)$ block of 20 pecks during testing. Chicks treated with 30 ng DHEA showed a tendency toward lower levels of errors in the first block of 20 pecks during testing, but as noted above, this trend did not reach significance (see Fig. 2).

Treatment had no effect on (a) latency to peck during training $\left(\mathrm{F}_{2,35}=0.66, \mathrm{P}=0.52\right)$, (b) total time to complete the training $\left(\mathrm{F}_{2.35}=0.31, \mathrm{P}=0.74\right)$, or $(\mathrm{c})$ total time spent pecking $\left(\mathrm{F}_{2.35}=1.57, \mathrm{P}=0.22\right.$; see Fig. 3A, 3B), but it significantly affected latency to peck during testing $\left(F_{2.35}=3.42, \quad P<0.05\right)$. Posthoc Tukey's HSD tests indicated that this result was due to the significantly reduced latency in chicks treated with $30 \mathrm{ng}$ DHEA as compared with matched saline-treated chicks $(P<0.05)$. There was no significant difference between saline- and $3 \mathrm{ng}$ DHEA-treated chicks in latency at test. The total time taken to complete 60 pecks $\left(\mathrm{F}_{2.35}=11.30, \mathrm{P}<0.01\right)$ was significantly different, however, indicating that the total amount of time spent in the testing cage differed between groups.

Both 3 and 30 ng DHEA-treated chicks spent less overall time in the experimental cage than did saline-treated chicks $(\mathrm{P}<0.01$ in each case). This result was not just a consequence of the reduced latency to peck, as was evident in both DHEAtreated groups (whereas reduced latency was evident only in the 30 ng-DHEA treated group). The total time taken to complete the 60 testing 


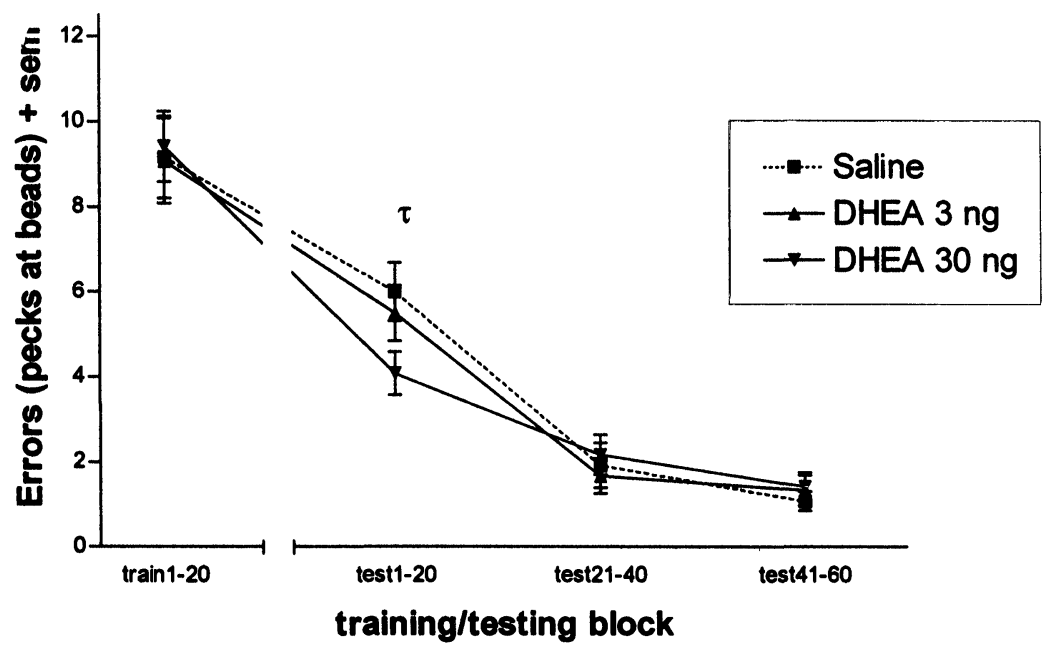

Fig. 2: Effects of a bilateral injection of DHEA (3 or $30 \mathrm{ng}$ in saline; upright or inverted triangles) or saline (squares) into the IMHV 5 min before training on the pecking accuracy of chicks during both training and testing. Data are analysed and presented as errors (pecks at beads) in each block of 20 pecks. $\tau$ indicates a trend towards statistical difference from the internal, salineinjected, control group and 3 ng DHEA-treated group, $0.10>P>0.05 . n=12$ for each group.

pecks (from first to last peck) was also significantly reduced in both 3 and 30 ng-DHEA treated chicks $\left(\mathrm{F}_{2.35}=13.52, \mathrm{P}<0.01\right)$. Both DHEA groups showed significantly reduced pecking times compared with saline-treated chicks $(P<0.01$ in both cases; see Fig. 3B). Thus, DHEA appears to reduce the time taken to complete the test without disrupting the overall accuracy of the responses.

\section{Experiment 3: Beadfloor testing chicks, very limited training}

Even with very limited training on the beadfloor (10 pecks), DHEA had no effect on accuracy in pecking during training (Fig. 4) when compared with saline or non-injected chicks. There was no significant difference between DHEA-treated, saline-treated or untreated chicks $(G=1.61, P=$ 0.45 ) in the number of errors in the training block of 10 pecks. Nor was there any significant effect of treatment on pecking in either the first $(\mathrm{G}=1.15$, $\mathrm{P}=0.56)$, second $(\mathrm{G}=0.03, \mathrm{P}=0.99)$ or third $(\mathrm{G}=2.28, \quad \mathrm{P}=0.32)$ block of 20 pecks during testing. Unlike chicks trained with 20 pecks, there was not even a tendency for chicks treated with 30 ng DHEA to show lower levels of errors in the first block of 20 pecks during testing. This result is, at least in part, due to the relatively high levels of variability shown by these chicks.

It is worth noting, however, that mean error scores of between 7 (saline) and 5.2 (DHEAtreated chicks) were seen in the first block of 20 pecks in testing. This, together with comparison of the testing data presented in Fig. 2 (where the average error scores in the first block of 20 pecks at test ranged from 6 in saline-treated chicks to 4 in DHEA-treated chicks) strongly suggests that 10 pecks is sufficient for chicks to acquire the visual categorization. 

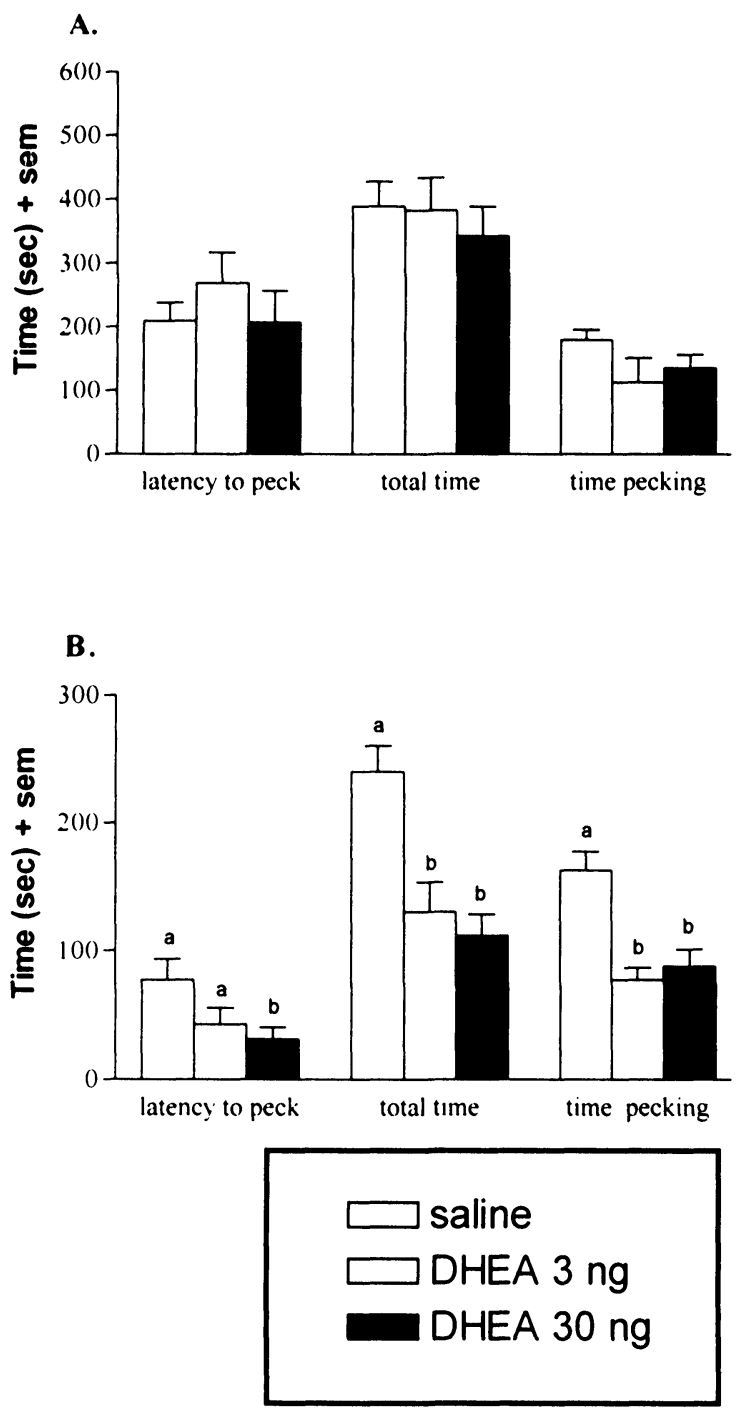

Fig. 3: Effects of a bilateral injection of DHEA (3 or $30 \mathrm{ng}$. shaded or solid bars) or saline (open bars) into the IMHV 5 min before training on the time taken to begin (latency) and complete (total time) training (A) and testing (B) on the beadfloor task. The total time spent pecking (total time minus latency) is also presented. Data are presented as mean \pm SEM. Bars with different letters indicate significant differences between treatments for that measure $\mathrm{P}<0.05$. $\mathrm{n}=12$ per group.

Treatment had no significant effect on latency to peck during training $\left(\mathrm{F}_{2.34}=0.006, \mathrm{P}>0.99\right)$, on the total time to complete the training $\left(\mathrm{F}_{2.35}=0.34\right.$,
$P=0.72)$ or on the total time spent pecking $\left(F_{2.35}\right.$ $=1.31, \mathrm{P}=0.28$; see Fig. 5, A and B). The lack of difference in any of these measured parameters between saline-treated, untreated, and DHEAtreated chicks (or saline- and 3 or 30 ng DHEAtreated chicks shown in Exp. 2) strongly suggests that treatment with DHEA does not alter learning by altering behaviors that are indirectly associated with task acquisition.

With very limited training, just as with limited training, treatment had a significant effect on latency to peck during testing $\left(\mathrm{F}_{2.35}=3.55\right.$, $P=0.04$ ). This result is clearly due to significantly reduced latency to peck in the DHEA-treated group. No difference occurred between saline-treated and untreated chicks, indicating that the stress assoc-iated with an intracerebral injection just before training is not sufficient to reduce latency at test $(\mathrm{P}>0.05)$ or accuracy at test—see below).

Despite an apparent reduction in the total time of testing and the time spent pecking in the DHEA-treated group (see Fig. 5) neither measure was statistically significant (total time: $F_{2.35}=2.25$, $\mathrm{P}=0.12$; time spent pecking $\mathrm{F}_{2.35}=0.91, \mathrm{P}=0.41$ ). This result is probably due primarily to the variability associated with these tests. The variability, however, was just as high in all groups (including untreated chicks) and might be related to the reduced training experience. Such variability is unlikely to be due to individual testing per se (no mirrors) as (note that the same scale in used in Figs. 3 and 5) testing individually without mirrors (but following an extended period of individual housing) did not appear to alter the average length of testing (but did, obviously, reduce the training times). Thus, DHEA appears to reduce the time taken to start the test, without disrupting the over-all accuracy of the responses for the time spent pecking-indicative of a possible memory enhancing effect of DHEA for this appetitive task. 


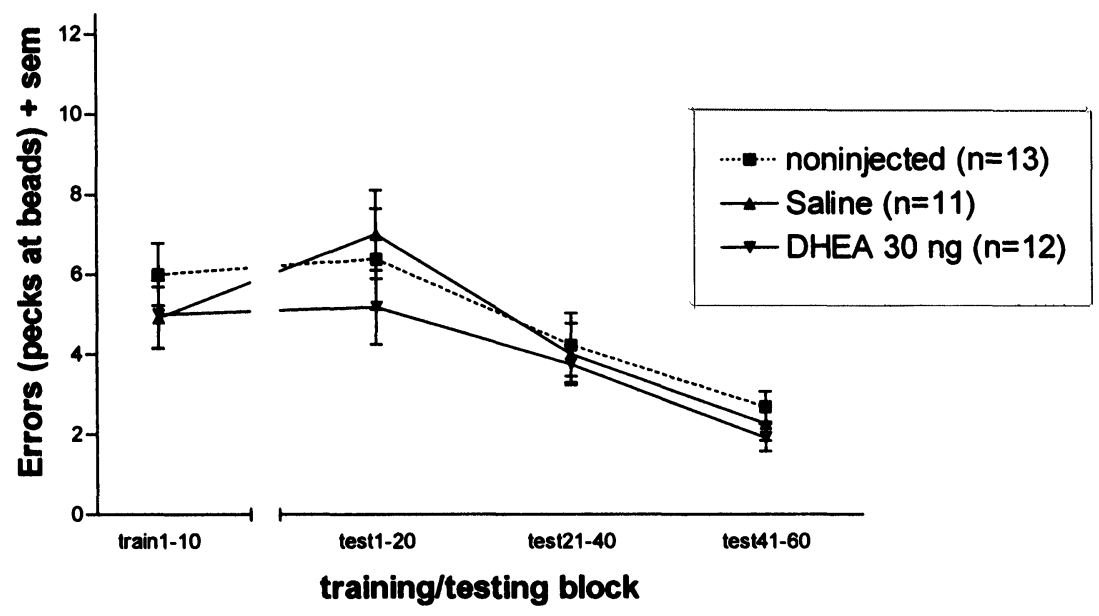

Fig. 4: Effects of a bilateral injection of DHEA ( $30 \mathrm{ng}$ in saline; inverted triangles) or saline (triangles) into the IMHV 5 min before training on the pecking accuracy of chicks during training and testing on the beadfloor task. Also included in this experiment is data from a non-injected group of chicks (squares). Data are analysed and presented as in Fig. 2. Note however that the training block was reduced to 10 pecks. $n$ values vary and are given in the legend key.

\section{Experiment 4: Open field and response to novel object tests}

Treatment with DHEA or saline, either 'pre' training ( $24 \mathrm{~h}$ before testing) or 'post' training (5 min before testing), did not selectively alter the responses of chicks in the open field test. Neither the number of squares crossed by the target chicks in each group $\left(\mathrm{F}_{3.23}=0.66, \mathrm{P}=0.59\right)$, nor the number of peeping bouts $\left(\mathrm{F}_{3.23}=1.07, \mathrm{P}=0.38\right)$ or latency to move out of the start square $\left(F_{3.23}=0.64\right.$, $P=0.60$ ) were significantly different between the groups. Four or 5 of the 6 chicks in each group defecated during the test $(\mathrm{G}=0.45, \mathrm{P}>0.50)$. Although the data showed a relatively high degree of variability (see Table 1), the response was not altered by treatment.

Similar results were obtained in the response to novel object tests. Again, the number of squares crossed by chicks did not appear to depend on their prior treatment $\left(\mathrm{F}_{3.23}=0.56, \mathrm{P}=0.65\right)$. Perhaps, not surprisingly, given the chicks previous experience in the open field apparatus, latency to move out of the start square was reduced in all groups. There was, however, no overall difference between the groups in latency to move from the start square $\left(\mathrm{F}_{3.23}=0.21, \quad \mathrm{P}=0.89\right) \quad$ or bouts of peeping $\left(\mathrm{F}_{3.23}=0.28, \mathrm{P}=0.84\right)$ in this test (see Table 1). None of the chicks approached or made contact with the novel object, and only two chicks spent time in the square adjacent to the novel object, negating useful analysis of the data. Interestingly, overall, chicks treated with DHEA defecated more often than saline-treated chicks did $(\mathrm{G}=6.28, \mathrm{P}<$ 0.05 ), although there was no difference between instances of defecation in chicks treated with DHEA either $24 \mathrm{hr}$ or $5 \mathrm{~min}$ before testing $(\mathrm{G}=$ $0.45, \mathrm{P}>0.50$ ).

\section{Experiment 5: Diffusion of DHEA throughout forebrain.}

The percentage of radioactivity retained in the whole brain is shown in Table 2. Approximately $20 \%$ of the radioactivity from DHEA was retained in the brain $5 \mathrm{~min}$ after injection, and it had almost 

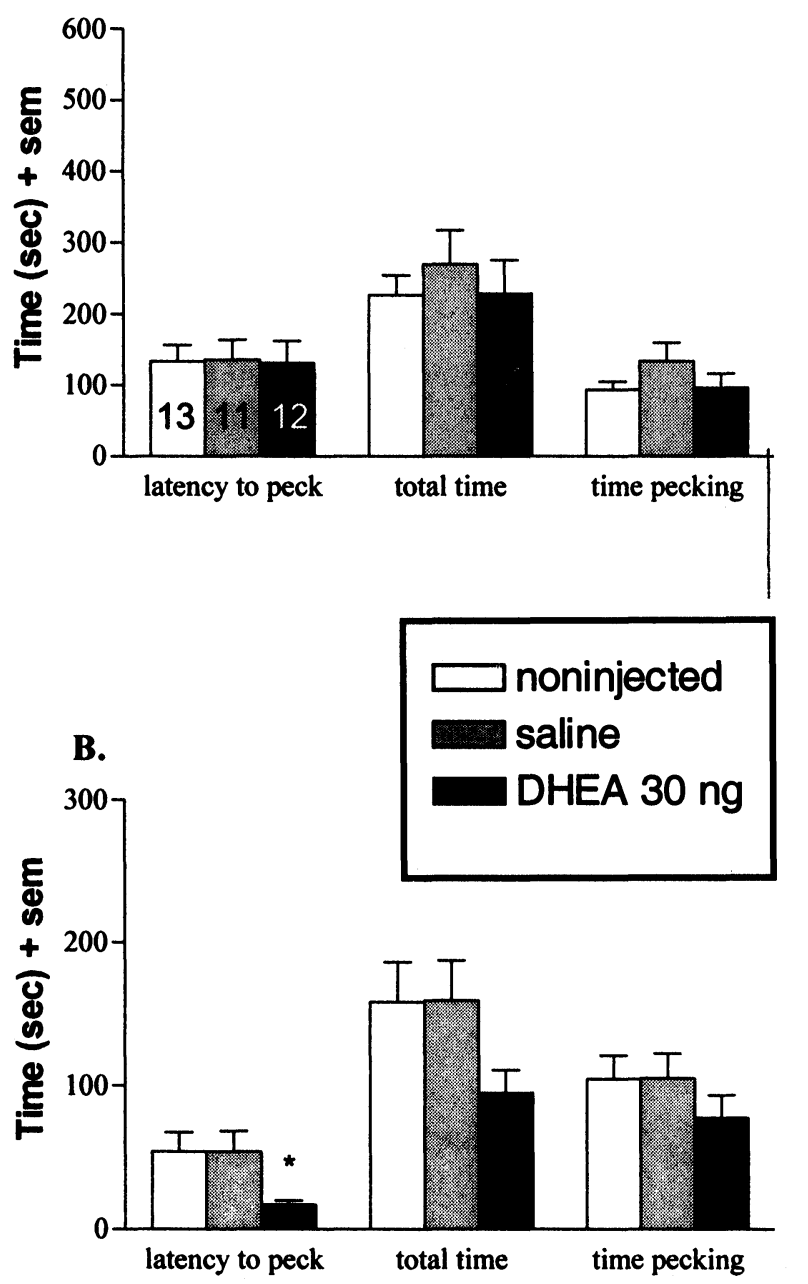

Fig. 5: Effects of a bilateral injection of DHEA (30 ng, solid bars) into the IMHV 5 min pretraining on latency to peck and total time spent completing training $(\mathrm{A})$ and testing (B) on the beadfloor task. The data are compared with those from chicks injected with saline (shaded bars) and a non-injested group of chicks (open bars). The total time spent pecking (total time minus latency) is also presented for training (A) and testing (B). Data are presented as in Fig. 3. * indicates significant differences between treatments on that measure $P<0.05$. $n$ values vary and are given in the appropriate bar.

disappeared by $1 \mathrm{hr}$ post-injection. Although the radioactivity decreased with time, the distribution within the IMHV and LPO was relatively constant. Approximately half the amount of total radio- activity retained within the brain is localized in the IMHV, whereas approximately $10 \%$ remains in the LPO (See Table 2).

\section{DISCUSSION}

The experiments described in this paper indicate that administration of exogenous DHEA has an enhancing effect on memory formation. The results confirm that memory retention in chicks trained on the 'weak' version of the passive avoidance task is enhanced by the administration of $30 \mathrm{ng}$ DHEA. This effect is unlikely to be due to nonspecific effects of DHEA on behavior (such as altered attention, activity or pecking behavior) because a pre-training injection of the neurosteroid did not alter the proportion of chicks in each group that reached criteria.

On the visual categorization task, the observation that administration of the higher dose of DHEA before training reduced latency to peck and total time of pecking without altering accuracy suggests the possibility that DHEA could enhance recall on a visual appetitive task as well. The data suggest that the effects of neurosteroids like DHEA are not limited to aversive or spatially oriented tasks, at least not in chicks. The effects of DHEA observed in 3 to 4 day old chicks also suggest that the role of DHEA is not limited to a very brief period of extreme plasticity in the chick (such as that described in day-old, 24 to $36 \mathrm{~h}$ chicks by Andrew [1991] and Rogers [1995]).

The report presented here is, to the best of our knowledge, the first on the use of limited training on the visual discrimination/categorization task as a means of demonstrating the enhancing effect of a neurochemical on recall. Although the 10-peck protocol seemed to show high levels of variationso high as to possibly blur enhancing effects of DHEA - the same pattern of results was evident with both the 20-peck and 10-peck protocols. 
TABLE 1

Behavioral data ${ }^{1}$ obtained in the open field and response to novel object tests

\begin{tabular}{|c|c|c|c|}
\hline Treatment & Squares crossed & Bouts peeping & latency \\
\hline DHEA 24 hr pretest & $15.2 \pm 1.49$ & $6.7 \pm 0.2$ & $81.0 \pm 26.6$ \\
Saline 24 hr pretest & $14.0 \pm 3.7$ & $3.5 \pm 3.5$ & $94.3 \pm 27.5$ \\
DHEA 5 min pretest & $19.7 \pm 4.0$ & $21.3 \pm 13.2$ & $50.0 \pm 23.8$ \\
Saline 5 min pretest & $15.2 \pm 2.5$ & $17.2 \pm 7.9$ & $64.5 \pm 17.1$ \\
\hline
\end{tabular}

${ }^{1}$ Expressed as mean \pm SEM

TABLE 2

Recovery and distribution of $\left[{ }^{3} \mathrm{H}\right] \mathrm{DHEA}$ in the chick brain ${ }^{\prime}$

\begin{tabular}{|c|c|c|c|}
\hline $\begin{array}{c}\text { Time after } \\
\text { injection (min) }\end{array}$ & $\begin{array}{c}\text { \% recovery in } \\
\text { brain }\end{array}$ & $\begin{array}{c}\text { \% of } \\
\text { radioactivity in } \\
\text { IMHV }\end{array}$ & $\begin{array}{c}\text { \% of } \\
\text { radioactivity in } \\
\text { LPO }\end{array}$ \\
\hline 5 & $24 \pm 2.2$ & $41 \pm 8$ & $13 \pm 4$ \\
15 & $9.7 \pm 0.8$ & $34 \pm 7$ & $11 \pm 1$ \\
30 & $5.1 \pm 1.9$ & $45 \pm 12$ & $12 \pm 4$ \\
60 & $1.4 \pm 0.9$ & $38 \pm 6$ & $15 \pm 5$ \\
\hline
\end{tabular}

\footnotetext{
'Data in the second column are expressed as a percentage of injected radioactivity. Data in columns 3 and 4 are expressed as percentage of total radioactivity measured in the whole brain.
}

The experiment examining diffusion of DHEA throughout the brain after intracerebral injection into the IMHV showed that only around $20 \%$ of the DHEA contained in the microsyringe was retained in the brain $5 \mathrm{~min}$ after injection, and almost all had disappeared by $1 \mathrm{~h}$ post-injection. The results presented here imply the following:

- the doses said to be administered into the IMHV in this and in other such studies (see also Mileusnic et al., 2000) are probably over- estimated, making difficult the accurate determination of the effective dose using behavioral pharmacological tests;

- DHEA is probably retained, unchanged, in the brain for no longer than $1 \mathrm{~h}$, which does not indicate whether it is metabolized to other steroids or if it diffuses away;

- the action of the neurosteroids injected into the IMHV might not be restricted only to this brain region but also occurs in the LPO, in the archistriatum, and in other surrounding areas. 
Our results mirror those of previous experiments conducted in our laboratory showing low recovery of tritiated substances injected into the IMHV (about 30\% with glutamate; Johnston, unpublished) and others in this field. Rosenzweig et al. (1991), who conducted a similar study with glutamate, enkephalin, and ouabain, also found a low percentage of retention, varying between $20 \%$ and $40 \%$, depending on the substance injected. Although this result clearly does not negate our behavioral findings, it does argue for caution in direct comparison of doses and the brain regions that are required for various learning tasks in different species based on the results of behavioral pharmacological experiments.

Migues et al. (2002) have suggested that the memory enhancing effect of DHEA might be related to the glucocorticoid-like effects of DHEA. Corticosterone has previously been shown to enhance at least the weak form of passive avoidance learning (Sandi \& Rose, 1994; Johnston \& Rose, 1998), and, although not investigated directly in the beadfloor, 'stress' or 'stress-like effects' have been implicated in altered consolidation of the pebble-floor (Rogers, 1995). DHEA can act via anti-glucocorticoid like mechanisms (Kalimi et al., 1994; see also Kimonides et al., 1999), whereas behavioral studies in adult male mice have suggested an anxiolytic effect of DHEA (Melchior \& Ritzmann, 1994; see also Frye \& Lacey, 1999).

The importance of the open field and response to novel object tests in batch-matched animals is that no significant effect of the drug (or injection) on these classic tests of possible fear- or stressrelated behaviors appears to be demonstrated. Such lack of effect supports the notion that DHEA may interact directly with the biochemical cascade associated with memory consolidation.

A great deal is known about the memory cascade associated with the consolidation of passive avoidance memory (Rose, 1991; 1995; 2000); less is known about the consolidation of categorization learning. Yet, both have been shown to involve similar stages of protein-synthesis dependency and fucosylation (Tiunova et al., 1996). Thus, it is possible that they might involve similar, if not the same, molecular/biochemical cascades. Certainly, some brain regions seem to be important for both processes. The IMHV in young chicks is crucial for the consolidation of passive avoidance learning, as well as for filial imprinting and possibly for the visual discrimination/categorization associated with learning to feed (Andrew, 1991). The relatively high amount of free DHEA in the IMHV has been taken as further support for a proposed role of DHEA in memory formation in the chick, possibly by a training-related stimulation of release of already synthesized DHEA into the extra-cellular space.

Various mechanisms could explain the effect of DHEA on memory retention. Such mechanisms include mediation through modulatory actions on neurotransmitter receptors like GABA and sigma receptors, which have been implicated in the mechanisms underlying at least passive avoidance learning (Clements \& Bourne, 1996; Maurice et al., 1998; Salinska et al., 1998). Alternatively, the effect of DHEA could be mediated by the classic genomic action of steroids (Rupprecht et al., 1996), facilitating the synthesis of proteins needed for long-term memory formation to occur. Although intracellular receptors have not yet been described for DHEA, it is possible that the compound is metabolized and acts on intracellular receptors (progesterone, testosterone, or corticoid receptors, for example) via one or more of its derivatives.

The effects of exogenous DHEA on recall could also occur via its action on neural cytoskeleton dynamics. DHEA has been shown to increase the number and connectivity of neurons in culture (Bologa et al., 1987), as well as increasing the length of the neurites containing the axonal marker Tau (Compagnone \& Mellon, 1998). Following strong passive avoidance training in chicks, memory-related changes are known to 
occur in synaptic and in dendritic numbers, dimensions, and morphology (Patel et al., 1988; Doubell \& Stewart, 1993), including changes in the connectivity of neurons in the IMHV region.

The mechanisms for DHEA action cited above are clearly not mutually exclusive and may well operate simultaneously in producing a memoryenhancing effect in chicks for appetitive, as well as for aversive conditioning. Although further studies are necessary to explore the precise route by which DHEA strengthens memory retention, our findings point to a significant regulatory role for DHEA, and presumably for related neurosteroids, in the normal mechanisms of experience-dependent neural plasticity. One of the few positive effects of the neurosteroids in human memory trials is that of DHEA on tasks involving visual attention in elderly adults (Wolf et al., 1998). Thus, DHEA may well constitute a potential therapeutic tool in the treatment of cognitive deficits.

\section{ACKNOWLEDGEMENTS:}

We are extremely grateful to Prof. S.P.R. Rose and to Dr R. Mileusnic for valuable comments on the manuscript and various discussions about the experiments contained in this paper. We also thank the anonymous referees for their valuable input into the final manuscript. We also acknowledge the Open University grant BR60-x-030 that funded this work.

\section{REFERENCES}

Akwa Y, Baulieu EE. 1999. Neurosteroids: behavioural aspects and physiological implications. J Soc Biol 193: 293-298.

Andrew RJ. 1991. Neural and behavioral plasticity: The use of the domestic chick as a model. New York, NY, USA: Oxford University Press.

Barbaccia ML, Concas A, Roscetti G, Bolacchi F, Mostallino MC, Purdy RH, Biggio G. 1996a. Stress-induced increase in brain neuroactive steroids: Antagonism by abecarnil. Pharmacol Biochem Behav 54: 205-210.

Barbaccia ML, Roscetti G, Trabucchi M, Mostallino MC, Concas A, Purdy RH, Biggio G. 1996b. Time-dependent changes in rat brain neuroactive steroid concentrations and $\mathrm{GABA}(\mathrm{A})$ receptor function after acute stress. Neuroendocrinology 63: $166-172$.

Baulieu EE. 1997. Neurosteroids: Of the nervous system, by the nervous system, for the nervous system. Rec Prog Horm Res 52: 1-32.

Baulieu EE, Robel P. 1996. Dehydroepiandrosterone and dehydroepiandrosterone sulfate as neuroactive neurosteroids. J Endocrinol 150: S221-S239.

Bologa L, Sharma E, Roberts E. 1987. Dehydroepiandosterone and its sulphate derivative reduce neuronal death and enhance astrocytic differentiation in brain cell cultures. J Neurosci Res 17: 225-234.

Burne THJ, Rose SPR. 1997. Effects of training procedure on memory formation using a weak passive avoidance learning paradigm. Neurobiol Learn Mem 68: 133-139.

Clements MP, Bourne, RC. 1996. Passive avoidance learning in the day-old chick is modulated by GABAergic agents. Pharmacol Biochem Behav 53: 629-634.

Cordero MI, Merino JJ, Sandi C. 1998. Correlational relationship between shock intensity and corticosterone secretion on the establishment and subsequent expression of contextual fear conditioning. Behav Neurosci 112: 885-891.

Compagnone NA, Mellon SH. 1998. Dehydroepiandrosterone: A potential signaling molecule for neocortical organization during development. Proc Natl Acad Sci USA 95: 4678-4683.

Corpechot C, Robel P, Axelson M, Sjovall J, Baulieu EE. 1981. Characterization and measurement of dehydroepiandosterone sulfate in the rat brain. Proc Natl Acad Sci USA 78: 4704-4707.

Corpechot C, Synguelakis M, Talha S, Axelson M, Sjovall J, Vihko R, et al. 1983. Pregnenolone and its sulphate ester in the rat brain. Brain Res 270: 119-125.

Crowe SF, Hamalainen M. 2001. Comparability of a single-trial passive avoidance learning task in the young chick across different laboratories. Neurobiol Learn Mem 75: 140-148.

Crowe SF, Ng KT, Gibbs ME. 1989. Memory formation processes in weakly reinforced learning. 
Pharmacol Biochem Behav 33: 881-887.

Demirgoren S, Majewska MD. Spivak CE, London ED. 1991. Receptor binding and electrophysiological effects of dehydroepiandosterone sulfate, an antagonist of the $\mathrm{GABA}_{\mathrm{A}}$ receptor. Neuroscience 45: $127-135$.

Doubell TP, Stewart MG. 1993. Short-term changes in the numerical density of synapses in the intermediate and medial hyperstriatum ventrale following one-trial passive avoidance training in the chick. J Neurosci 13: 2230-2236.

Farkas L, Crowe SF. 2000. The role of the benzodiazepine-GABA system in the memory processes of the day-old chick. Pharmacol Biochem Behav 65: 223-231.

Flood JF, Smith GE, Roberts E. 1988. Dehydroepiandrosterone and its sulfate enhance memory retention in mice. Brain Res 447: 269-278.

Frye CA, Lacey EH. 1999. The neurosteroids DHEA and DHEAS may influence cognitive performance by altering affective state. Physiol Behav 66: 8592.

Horn G. 1991. Cerebral function and behavior investigated through a study of filial imprinting, Cambridge, UK: Cambridge University Press.

Izquierdo I, Medina JH. 1997. Memory formation: The sequence of biochemical events in the hippocampus and its connection to activity in other brain structures. Neurobiol Learn Mem 68: 285316.

Johnston ANB, Clements MP, Rose SPR. 1999. Role of brain-derived neurotrophic factor and presynaptic proteins in passive avoidance learning in day-old domestic chicks. Neuroscience 88: 1033 1042.

Johnston ANB, Dodd P, Rogers LJ. 1995. [ [ H]MK801 binding asymmetry in the IMHV region of the forebrain of dark-reared chicks is reversed by imprinting. Brain Res Bull 37: 5-8.

Johnston ANB, Rose SPR. 1998. Isolation-stressinduced facilitation of passive avoidance memory in the day-old chick. Behav Neurosci 112: 929936.

Kalimi M, Shafagoj Y, Loria R, Padgett D, Regelson W. 1994. Anti-glucocorticoid effects of dehydroepi-androsterone (DHEA). Mol Cell Biochem 131: 99-104.

Kimonides VG, Spillantini MG, Sofroniew MV, Fawcett JW, Herbert J. 1999. Dehydroepiandrosterone antagonizes the neurotoxic effects of corticosterone and translocation of stressactivated protein kinase 3 in hippocampal primary cultures. Neuroscience 89: 429-436.

Lanthier A, Patwardhan VV. 1986. Sex steroids and 5en-3-beta-hydroxysteroids in specific regions of the human brain and cranial nerves. J Steroid Biochem 25: 445-449.

McGaugh JL. 1989. Involvement of hormonal and neuromodulatory mechanisms in the regulation of memory storage. Ann Rev Neurosci 12: 255-287.

Majewska MD, Demirgoren D, Spivak CE, London ED. 1990. The neurosteroid dehydroepiandosterone sulfate is an allosteric antagonist of the $\mathrm{GABA}_{\mathrm{A}}$ receptor. Brain Res 526: 143-146.

Maurice T, Su TP, Privat A. 1998. Sigma(1) receptor agonists and neurosteroids attenuate B25-35-amyloid peptide-induced amnesia in mice through a common mechanism. Neuroscience 83: 413-428.

Melchior CL, Ritzmann RF. 1996. Neurosteroids block the memory-impairing effects of ethanol in mice. Pharmacol Biochem Behav 53: 51-56.

Melchior CL, Ritzmann RF. 1994. Dehydroepiandosterone is an anxiolytic in mice on the plus maze. Pharmacol Biochem Behav 47: 437-441.

Migues, PV, Johnston ANB, Rose SPR. 2002. Dehydroepiandosterone and its sulphate enhance memory retention in day-old chicks. Neuroscience 109: 243-251.

Mileusnic R, Lancashire CL, Johnston ANB, Rose SPR. 2000. APP is required during an early phase of memory formation. Eur $\mathrm{J}$ Neurosci 12: $4487-$ 4495

Monnet FP, Mahe V, Robel P, Baulieu EE. 1995. Neurosteroids, via sigma-receptors, modulate the $\left[{ }^{\mathrm{H} 3}\right]$ norepinephrine release evoked by $\mathrm{N}$-methylD-aspartate in the rat hippocampus. Proc Natl Acad Sci USA 92: 3774-3778.

$\mathrm{Ng} \mathrm{K}$, Gibbs ME, Crowe SF, Sedman GL, Hua F, Zhao W, et al. 1992. Molecular mechanisms of memory formation. Mol Neurobiol 5: 333-350.

Patel SN, Rose SPR, Stewart MG. 1988. Training induced dendritic spine density changes are specifically related to memory formation processes in the chick, Gallus-Domesticus. Brain Res 463: 168-173.

Reddy DS, Kulkarni SK. 1998a. The effects of neurosteroids on acquisition and retention of a modified passive-avoidance learning task in mice. Brain Res 791: 108-116.

Reddy DS, Kulkarni SK. 1998b. Possible role of nitric 
oxide in the nootropic and antiamnesic effects of neurosteroids on ageing- and dizocilpine-induced learning impairment. Brain Res 799: 215-229.

Robel P, Baulieu EE. 1995. Neurosteroids: Biosynthesis and function. Critic Rev Neurobiol 9: 383-394.

Robel P, Schumacher M, Baulieu EE. 1999. Neurosteroids: From definition and biochemistry to physiopathologic function. In: Baulieu EE, Robel P, Schumacher M, eds, Neurosteroids. A New Regulatory Function in the Nervous System, New Jersey, USA: Humana Press; 1-26.

Roberts E, Bologa L, Flood JF, Smith GE. 1987. Effects of dehydroepiandrosterone and its sulfate on brain tissue in culture and on memory in mice. Brain Res 406: 357-362.

Rogers LJ. 1995. The Development of Brain and Behavior in the Chicken. Wallingford, Connecticut, USA: T. CAB International.

Rose SPR. 1991. How chicks make memories-the cellular cascade from c-Fos to dendritic remodelling. Trends Neurosci 14: 390-397.

Rose SPR. 1995. Glycoproteins and memory formation. Behav Brain Res 66: 73-78.

Rose SPR. 2000. God's organism? The chick as a model system for memory studies. Learn Mem 7: 1-17.

Rose SPR, Stewart MG. 1999. Cellular correlates of stages of memory formation in the chick following passive avoidance training. Behav Brain Res 98: 237-243.

Rosenzweig MR, Bennet EL, Martinez JL, Beniston D, Colombo PJ, Lee DW, et al. 1991. Stages of memory formation in the chick: findings and problems. In: Andrew RJ, ed, Neural and Behavioural Plasticity: The Use of the Domestic Chick as a Model. New York, NY, USA: Oxford University Press; 395-418.

Rupprecht R, Berning B, Hauser CAE, Holsboer F,
Reul JP. 1996. Steroid receptor-mediated effects of neuroactive steroids: Characterization of structure-activity relationship. Eur J Pharmacol 303: 227-234.

Salinska E, Bourne RC, Rose SPR. 1998. NMDA and non-NMDA glutamate receptors mediate calcium fluxes related to memory formation after passive avoidance task in day-old chicks. Eur J Neurosci 10: 5408 .

Sandi C, Rose SPR. 1994. Corticosterone enhances long-term retention in one-day-old chicks trained in a weak passive-avoidance learning-paradigm. Brain Res 647: 106-112.

Sokal R., Rohlf FJ. 1981. Biometry: The Principles and Practice of Statistics in Biological Research. New York, NY, USA: W.H. Freeman \& Co.

Tiunova A, Anokhin K, Rose SPR. 1998. Two critical periods of protein and glycoprotein synthesis in memory consolidation for visual categorization learning in chicks. Learn Mem 4: 401-410.

Tiunova A, Anokhin K, Rose SP, Mileusnic R. 1996. Involvement of glutamate receptors, protein kinases, and protein synthesis in memory for visual discrimination in the young chick. Neurobiol Learn Mem 65: 233-234.

Tsutsui K, Yamazaki T. 1995. Avian neurosteroids .1. Pregnenolone biosynthesis in the quail brain. Brain Res 678: 1-9.

Vallee M, Mayo W, Koob GF, LeMoal M. 2001. Neurosteroids in learning and memory processes. Int Rev Neurobiol 46: 273-320.

Wolf OT, Kudielka BM, Hellhammer DH, Hellhammer J, Kirschbaum C. 1998. Opposing effects of DHEA replacement in elderly subjects on declarative memory and attention after exposure to a laboratory stressor. Psychoneuroendocrinology 23: $617-629$. 

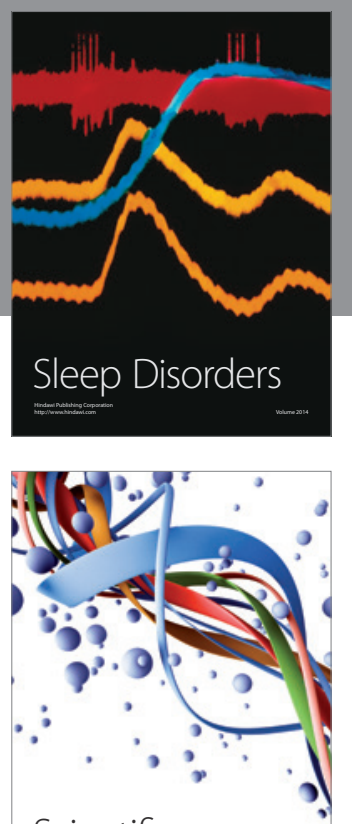

Scientifica
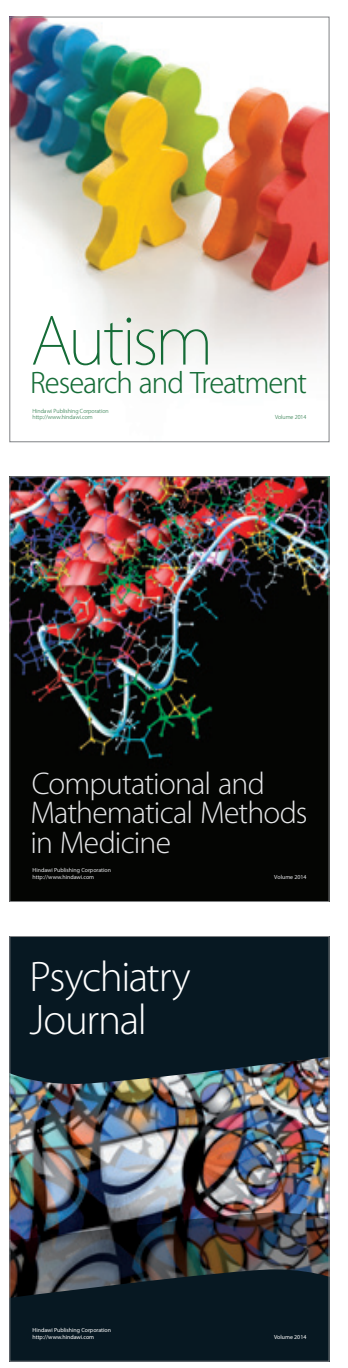
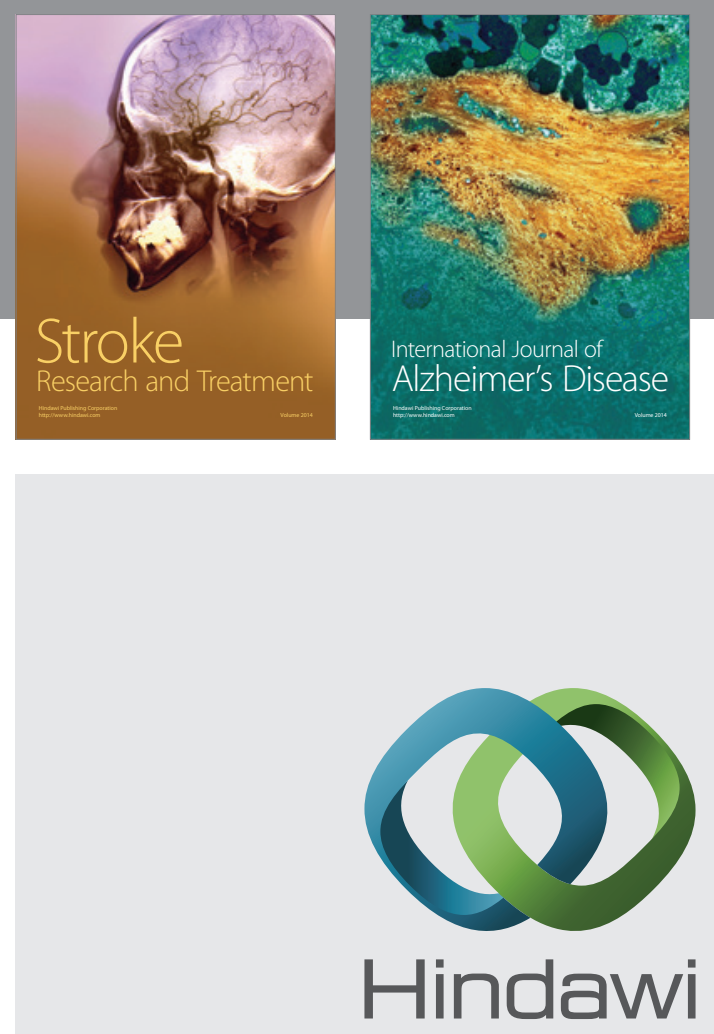

Submit your manuscripts at

http://www.hindawi.com
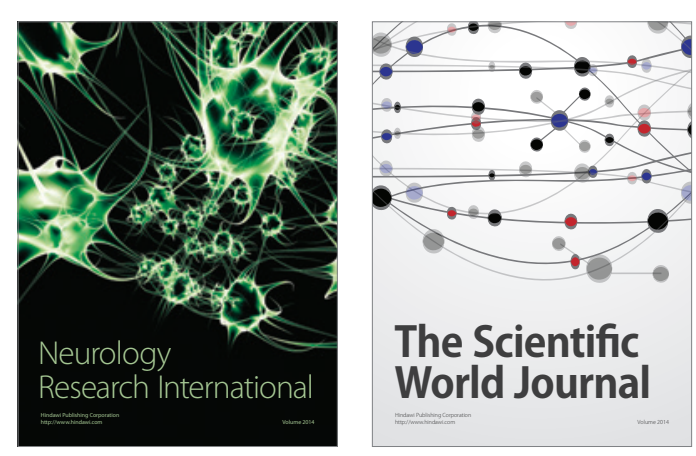

The Scientific World Journal

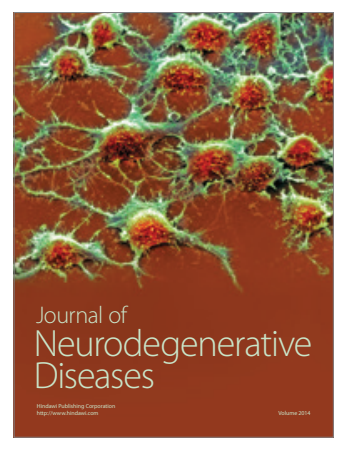

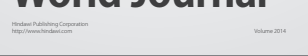

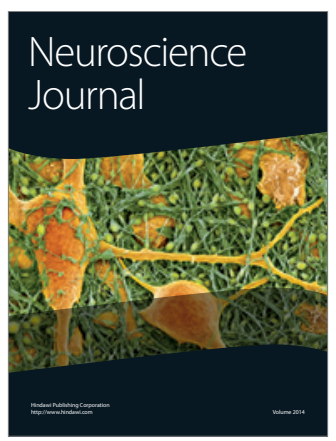

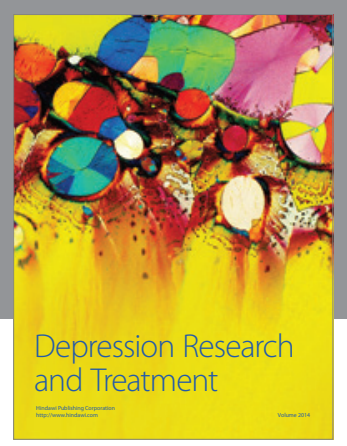
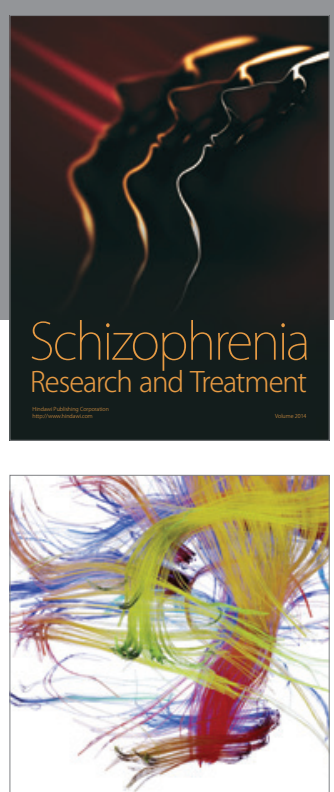

Brain Science

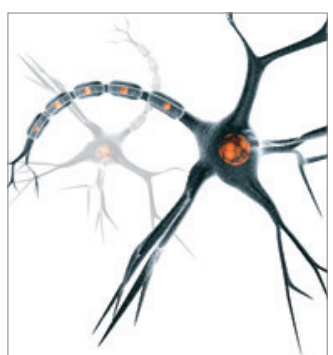

Neural Plasticity
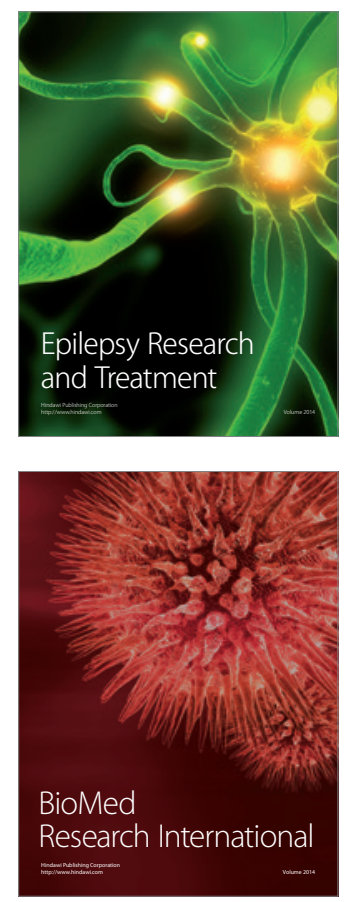

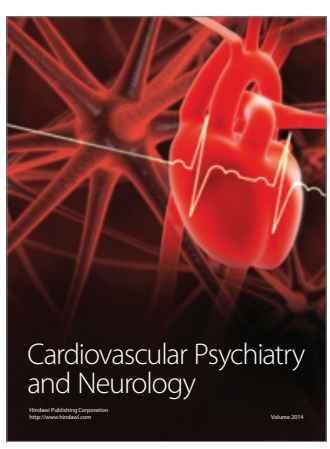

Parkinson's

Disease
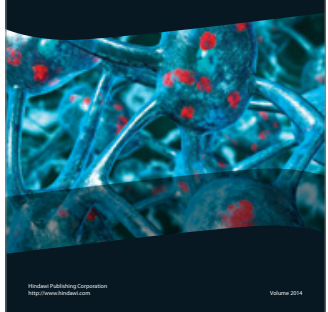